\title{
Heavy Exotic Molecules
}

\author{
Yizhuang Liu* and Ismail Zahed† \\ Department of Physics and Astronomy \\ State University of New York, Stony Brook, NY 11794-3800
}

\begin{abstract}
We briefly review the formation of pion-mediated heavy-light exotic molecules with both charm and bottom, under the general strictures of chiral and heavy quark symmetries. The charm isosinglet exotic molecules with $J^{P C}=1^{++}$binds, which we identify as the reported neutral $X(3872)$. The bottom isotriplet exotic with $J^{P C}=1^{+-}$binds, and is identified as a mixed state of the reported charged exotics $Z_{b}^{+}(10610)$ and $Z_{b}^{+}(10650)$. The bound bottom isosinglet molecule with $J^{P C}=1^{++}$is a possible neutral $X_{b}(10532)$ to be observed.
\end{abstract}

To appear in Nuclei, Quarks, Stars -memorial dedicated to Gerry Brown,

Eds. J.W. Holt, T.T.S. Kuo, K.K. Phua, M. Rho and I. Zahed, World Scientific (2016).

*Electronic address: yizhuang.liu@stonybrook.edu

${ }^{\dagger}$ Electronic address: ismail.zahed@stonybrook.edu 


\section{INTRODUCTION}

A decade ago, the BaBar collaboration [1] and the CLEOII collaboration [2] have reported narrow peaks in the $D_{s}^{+} \pi^{0}(2317)$ and the $D_{s}^{*+} \pi^{0}(2460)$ in support of predictions from chiral and heavy-quark symmetry [3] 5 . The heavy-light multiplet $\left(0^{-}, 1^{-}\right)=\left(D, D^{*}\right)$ has a chiral partner $\left(0^{+}, 1^{+}\right)=\left(\tilde{D}, \tilde{D}^{*}\right)$ that is about one consituent mass heavier [3, 4]. More recently, the Belle collaboration [6] and the BESIII collaboration [7] have reported new multiquark exotics, outside the standard quark model classification. A key source for these exotics is $\Upsilon(10860)$ and its closeness to the $B \bar{B}^{*} \pi$ (10744) and $B^{*} \bar{B}^{*} \pi$ (10790) tresholds. The smallness of the phase space for the pion decay of $\Upsilon(10860)$ suggests that the decay process is slow, involving a molecular configuration on the way out. Heavy exotic molecules have been reported, such as the neutral $X(3872)$ and the charged $Z_{c}(3900)^{ \pm}$and $Z_{b}(10610)^{ \pm}$. More of these exotics are expected to be unravelled by the DO collaboration at Fermilab [8], and the LHCb collaboration at Cern [9].

Theoretical arguments have predicted the possibility of molecular bound states involving heavy-light charm and bottom mesons through pion exchang [10, 11]. Since, a number of molecular estimates were made by many [11 16]. Non-molecular heavy exotics were also propodsed using constituent quark models [17], heavy solitonic baryons [18, 19], instantons [20] and QCD sum rules [21].

In this contribution, we briefly review our recent analysis of the molecular configurations with heavy-light charm and bottom mesons and their chiral partners, under the general constraints of chiral and heavy-quark symmetry [22]. In section 2, we outline the heavylight effective action in leading order involving the $\left(0^{ \pm}, 1^{ \pm}\right)$multiplets, and formulate the non-relativistic bound state problem in the $J=1$ channel. The results are summarized in section 3. Our conclusions are in section 4.

\section{MOLECULES}

The leading part of the heavy-light Lagrangian for the charmed multiplet $\left(0^{-}, 1^{-}\right)$with pions reads [3, 5] 


$$
\begin{aligned}
\mathcal{L} \approx & +2 i\left(\bar{D} \partial_{0} D+\overline{\boldsymbol{D}} \cdot \partial_{0} \boldsymbol{D}\right)-\Delta m_{D} \bar{D} D-\Delta m_{\boldsymbol{D}} \overline{\boldsymbol{D}} \boldsymbol{D} \\
& +i \frac{g_{H}}{f_{\pi}} \operatorname{Tr} \partial_{i} \pi\left(D_{i} D^{\dagger}-D D_{i}^{\dagger}+\epsilon_{i j k} D_{k} D_{j}^{\dagger}\right)
\end{aligned}
$$

with $\Delta m_{i}=m_{i}-m_{C}$ of the order of a quark constituent mass. The leading part of the heavy-light chiral doublers Lagrangian for the charmed $\left(0^{+}, 1^{+}\right)$multiplet with pions reads [3]

$$
\begin{aligned}
\tilde{\mathcal{L}} \approx \quad & +2 i\left(\overline{\tilde{D}} \partial_{0} \tilde{D}+\tilde{\tilde{\boldsymbol{D}}} \cdot \partial_{0} \tilde{\boldsymbol{D}}\right)-\Delta m_{\tilde{D}} \overline{\tilde{D}} \tilde{D}-\Delta m_{\tilde{\boldsymbol{D}}} \tilde{\tilde{\boldsymbol{D}}} \tilde{\boldsymbol{D}} \\
& +i \frac{g_{H}}{f_{\pi}} \operatorname{Tr} \partial_{i} \pi\left(i\left(\tilde{D}_{i} \tilde{D}^{\dagger}+\tilde{D} \tilde{D}_{i}^{\dagger}\right)+\epsilon_{i j k} \tilde{D}_{k} \tilde{D}_{j}^{\dagger}\right)
\end{aligned}
$$

with again $\Delta m_{\tilde{i}}=m_{\tilde{i}}-m_{C}$ of the order of a quark constituent mass. The $\left(0^{+}, 1^{+}\right)$multiplet mixes with the $\left(0^{-}, 1^{-}\right)$by chiral symmetry [3, 4 ]

$$
\delta \mathcal{L}=\frac{g_{H G}}{f_{\pi}} \operatorname{Tr} \partial_{0} \pi\left(\tilde{D}_{i}^{\dagger} D_{i}-i \tilde{D}^{\dagger} D+\text { c.c. }\right)
$$

The molecular exotics of the type $D \bar{D}^{*}$, follows from (1) through one-pion exchange. The non-relativistic character of the molecules yield naturally to a Hamiltonian description. Let $D_{0 \overline{0}}(\boldsymbol{r})$ denote the wave function of the molecular scalar, and $\bar{Y}_{0 \bar{i}}(\boldsymbol{r})$ and $Y_{i \overline{0}}(\boldsymbol{r})$ denote the wavefunctions of the molecular vectors, and $T_{i \bar{j}}(\boldsymbol{r})$ the wavefunction of the molecular tensors. Using (14) for the 2-body interactions, we have

$$
\begin{aligned}
& (V T)_{k \bar{l}}=C \epsilon_{k i m} \epsilon_{\overline{l j} n} \partial_{m n} V(r) T_{i \bar{j}} \\
& (V T)_{0 \overline{0}}=C \partial_{i \bar{j}} V T_{i \bar{j}} \\
& (V \bar{Y})_{k \overline{0}}=-C \partial_{k} \partial_{\bar{j}} V(r) \bar{Y}_{0 \bar{j}} \\
& (V T)_{0 \bar{k}}=C \epsilon_{\bar{k} \bar{l} j} \partial_{i} \partial_{j} V(r) T_{i \bar{l}} \\
& (V T)_{\overline{0} k}=C \epsilon_{k l j} \partial_{\bar{i}} \partial_{j} V(r) T_{\bar{l}}
\end{aligned}
$$

with the isospin factor 


$$
C=\boldsymbol{I}_{\mathbf{1}} \cdot \boldsymbol{I}_{\mathbf{2}}=\left(\left.\frac{1}{4}\right|_{I=1},-\left.\frac{3}{4}\right|_{I=0}\right)
$$

Here $V(r)$ is the regulated one-pion exchange using the standard monopole form factor by analogy with the pion-nucleon form factor [23]. It is defined with a core cutoff $\Lambda \gg$ $m_{\pi}[11,23]$

$$
V(r)=\left(\frac{g_{H}}{f_{\pi}}\right)^{2} \frac{1}{4 \pi}\left(\frac{e^{-m_{\pi} r}}{r}-\frac{e^{-\Lambda r}}{r}-\left(\Lambda^{2}-m_{\pi}^{2}\right) \frac{e^{-\Lambda r}}{2 \Lambda}\right)
$$

Throughout, we will use $g_{H}=0.6$ [3, 4] and $\Lambda=1 \mathrm{GeV}$. The choice of $\Lambda$ is the major uncertainty in the molecular analysis. The one-pion exchange in (4) induces a D-wave admixture much like in the deuteron as a proton-neutron molecule [23]. It is very different from one-gluon exchange in heavy quarkonia [17].

The pertinent projections onto the higher $J^{P C}$ channels of the molecular wavefunctions in (4) require the use of both vector and higher tensor spherical harmonics [24, 25]. For $J=1$, we will use the explicit forms quoted in [25] with the ${ }^{S} L_{J}$ assignment completly specified. For the $\left(1^{\mp}, 0^{\mp}\right)$ multiplets, there are 4 different $1^{P C}$ sectors

$$
\begin{aligned}
1^{++}: & T_{i \bar{j}}^{2,2}\left({ }^{5} D_{1}\right), Y_{i}^{0+}\left({ }^{3} S_{1}\right), Y_{i}^{2+}\left({ }^{3} D_{1}\right) \\
1^{--}: & T_{i \bar{j}}^{0,1}\left({ }^{1} P_{1}\right), T_{i \bar{j}}^{2,1}\left({ }^{5} P_{1}\right), T_{i \bar{j}}^{2,3}\left({ }^{5} F_{1}\right), \\
& Y_{i}^{1-}\left({ }^{3} P_{1}\right), D^{1}\left({ }^{1} P_{1}^{\prime}\right) \\
1^{+-}: & T_{i \bar{j}}^{1,0}\left({ }^{3} S_{1}\right), T_{i \bar{j}}^{1,2}\left({ }^{3} D_{1}\right), Y_{i}^{0-}\left({ }^{3} S_{1}^{\prime}\right), Y_{i}^{2-}\left({ }^{3} D_{1}^{\prime}\right) \\
1^{-+}: & T_{i \bar{j}}^{1,1}\left({ }^{3} P_{1}\right), Y_{i}^{1+}\left({ }^{3} P_{1}^{\prime}\right)
\end{aligned}
$$

The normalized tensor harmonics are detailed in [22, 25].

\section{RESULTS}

In Fig. 1 we show the the radial components (upper part) and percentage content (lower part) of the bound isosinglet charm wavefunction with energy $E=3.867 \mathrm{GeV}$, versus $r$ in units of $\Lambda=1 \mathrm{GeV}$. The intra-coupling between the $\left(0^{-}, 1^{-}\right)$and $\left(0^{+}, 1^{+}\right)$multiplet 

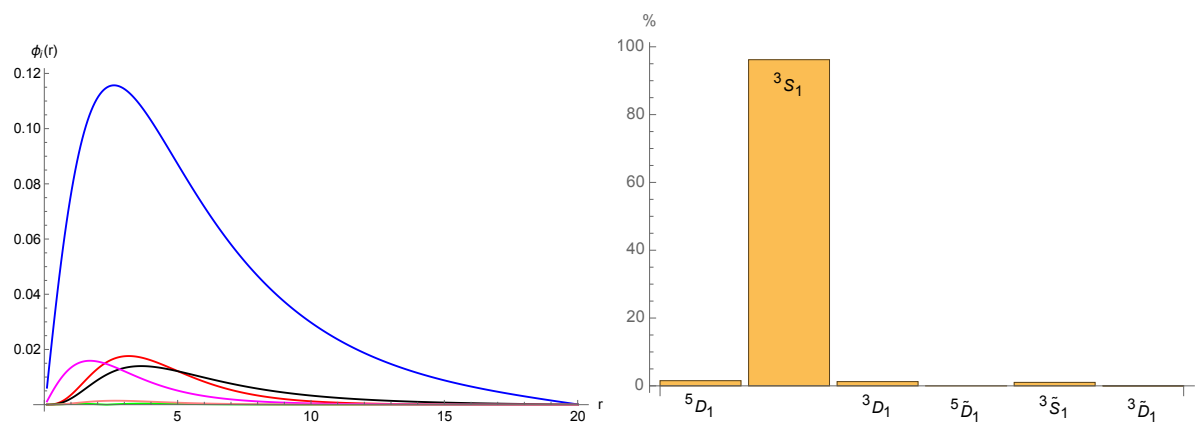

FIG. 1: $J^{P C}=1^{++}$: radial wavefunctions (upper plot) and percentage content (lower plot) for the charm isosinglet exotic state $(C=-3 / 4)$.

causes the chiral partners $\tilde{D} \overline{\tilde{D}}^{*}$ to unbind. The ${ }^{S} L_{J}$ assignments referring to the $\left(0^{-}, 1^{-}\right)$ multiplet, and the ${ }^{S} \tilde{L}_{J}$ assignments referring to the $\left(0^{+}, 1^{+}\right)$multiplet, are those listed in (7). The mixing results in a stronger binding in this channel wich is mostly an isosinglet ${ }^{1} S_{3}$ contribution in the $\left(1^{-}, 0^{-}\right)$multiplet with almost no D-wave admixture. This molecular state carries $J^{P C}=1^{++}$. It is chiefly an isosinglet $D \bar{D}^{*}$ molecule, which we identify as the reported exotic $X(3872)$.
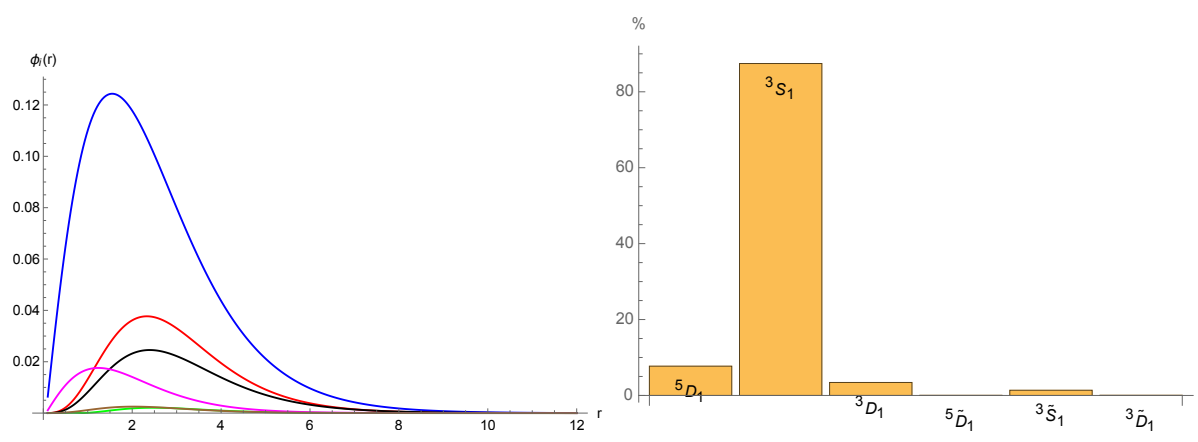

FIG. 2: $J^{P C}=1^{++}$: radial wavefunctions (upper plot) and percentage content (lower plot) for the bottom isosinglet exotic state $(C=-3 / 4)$.

In Fig. 2 we show the the radial components (upper part) and percentage content (lower part) of the bound isosinglet bottom wavefunction with energy $E=10.532 \mathrm{GeV}$, versus $r$ in units of $\Lambda=1 \mathrm{GeV}$. Again, the ${ }^{S} L_{J}$ and ${ }^{S} \tilde{L}_{J}$ assignments refer to the $\left(0^{ \pm}, 1^{ \pm}\right)$multiplets respectively, as defined in $(7)$. The $1^{++}$mixed bound state is mostly a $B \bar{B}^{*}\left({ }^{3} S_{1}\right)$ molecule. A comparison of Fig. 1 to Fig. 2 shows that this neutral bottom molecular state is the mirror analogue of the neutral charm molecular state or $X_{b}(10532)$, yet to be reported. 
In Fig. 3 we show the the radial components (upper part) and percentage content (lower part) of the bound isosinglet bottom wavefunction with energy $E=10.592 \mathrm{GeV}$, versus $r$ in units of $\Lambda=1 \mathrm{GeV}$. From the assignments given in (7), it follows that $1^{+-}$is a mixed isotriplet $B \bar{B}^{*}\left({ }^{3} S_{1}^{\prime}\right)$ molecules, with a small admixture of $B^{*} \bar{B}^{*}\left({ }^{3} S_{1}^{\prime}\right)$ molecule. This molecule is an admixture of the reported states $Z_{b}^{+}(10610)$ and $Z_{b}^{+}(10650)$.
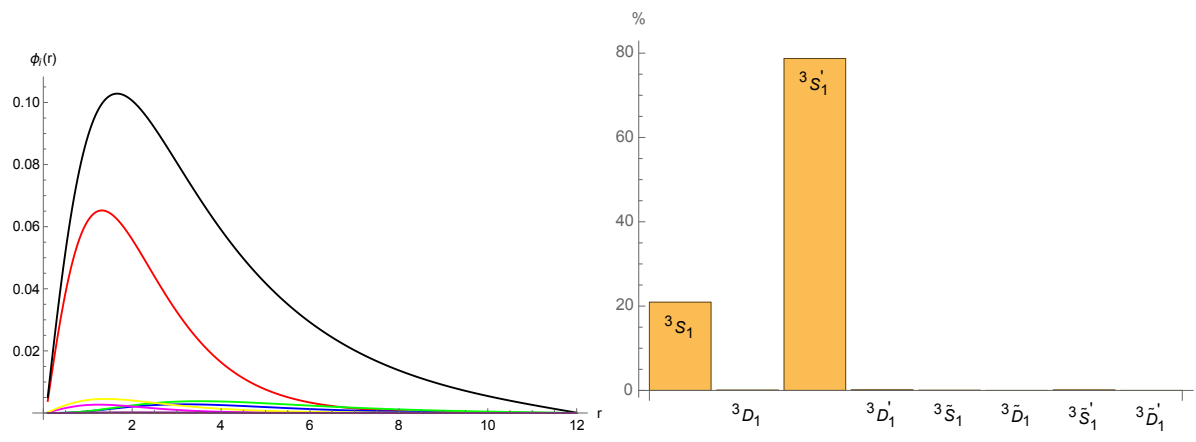

FIG. 3: $J^{P C}=1^{+-}$: radial wavefunctions (upper plot) and percentage content (lower plot) for the bottom isotriplet exotic state $(C=+1 / 4)$.

\section{CONCLUSIONS}

We have briefly reported on the molecular states of doubly heavy mesons mediated by one-pion exchange for both the chiral parteners $\left(0^{ \pm}, 1^{ \pm}\right)$as a coupled channel problem, recently discussed in [22]. The analysis complements and extends those presented in [11-16] by taking into account the strictures of chiral and heavy quark symmetry, and by retaining most coupled channels between the $\left(0^{-}, 1^{-}\right)$multiplet and its chiral partner $\left(0^{+}, 1^{+}\right)$. The key aspect of this coupling is to cause the molecules in the $\left(0^{-}, 1^{-}\right)$multiplet to bind about twice more, and the molecules in the $\left(0^{+}, 1^{+}\right)$multiplet to unbind. The charm isosinglet exotic molecules with $J^{P C}=1^{++}$is strictly bound for a pion-exchange cutoff $\Lambda=1 \mathrm{GeV}$. This state is identified with the reported isosinglet exotic $\mathrm{X}(3872)$ which in our case is mostly an isosinglet $D \bar{D}^{*}$ molecule in the ${ }^{1} S_{0}$ channel with no D-wave admixture. The attraction in the isotriplet channel with $J^{P C}=1^{+-}$is too weak to bind the $D \bar{D}^{*}$ compound, suggesting that the reported isotriplet $Z_{C}(3900)^{ \pm}$is at best a treshold enhancement. The $Y(4260), Y(4360)$ and $Y(4660)$ may point to the possibility of their constituents made of excited $\left(D_{1}, D_{2}\right)$ heavy mesons and their chiral partners [3, 26]. The isotriplet bottom exotic molecule with 
$J^{P C}=1^{+-}$which we have identified with the pair $Z_{b}^{+}(10610)$ and $Z_{b}^{+}(10650)$, which is a mixed state in our analysis. The isosinglet bottom exotic molecule with $J^{P C}=1^{++}$is a potential candidate for $X_{b}(10532)$, yet to be measured.

\section{TRIBUTE TO GERRY BROWN}

Ismail Zahed: Gerry has been a mentor and a colleague to me for the past three decades. He has taught many of us a great deal of how to approach physics from the bottom line point of view and encouraged us to pursue freely our physics interests, always in the quest of tangible results. Most of us here at Stony Brook and many elsewhere, owe so much to Gerry scientific leadership and personal friendship. We hope to pass his legacy to our students and collaborators.

\section{ACKNOWLEDGEMENTS}

This work was supported by the U.S. Department of Energy under Contract No. DEFG-88ER40388. 
[1] B. Aubert et al. [BaBar Collaboration], Phys. Rev. Lett. 90, 242001 (2003) hep-ex/0304021].

[2] D. Besson et al. [CLEO Collaboration], Phys. Rev. D 68, 032002 (2003) Erratum: [Phys. Rev. D 75, 119908 (2007)] hep-ex/0305100].

[3] M. A. Nowak, M. Rho and I. Zahed, Phys. Rev. D 48, 4370 (1993) hep-ph/9209272]; M. A. Nowak, M. Rho and I. Zahed, Acta Phys. Polon. B 35, 2377 (2004) hep-ph/0307102.

[4] W. A. Bardeen and C. T. Hill, Phys. Rev. D 49 (1994) 409 hep-ph/9304265; W. A. Bardeen, E. J. Eichten and C. T. Hill, Phys. Rev. D 68, 054024 (2003) hep-ph/0305049.

[5] N. Isgur and M. B. Wise, Phys. Rev. Lett. 66 (1991) 1130; A. V. Manohar and M. B. Wise, "Heavy quark physics," Camb. Monogr. Part. Phys. Nucl. Phys. Cosmol. 10, 1 (2000).

[6] I. Adachi [Belle Collaboration], arXiv:1105.4583 [hep-ex]; A. Bondar et al. [Belle Collaboration], Phys. Rev. Lett. 108, 122001 (2012) arXiv:1110.2251 [hep-ex]].

[7] M. Ablikim et al. [BESIII Collaboration], Phys. Rev. Lett. 110, 252001 (2013) arXiv:1303.5949 [hep-ex]].

[8] V. M. Abazov et al. [D0 Collaboration], arXiv:1602.07588 [hep-ex]].

[9] R. Aaij et al. [LHCb Collaboration], arXiv:1606.07895 [hep-ex]; R. Aaij et al. [LHCb Collaboration], arXiv:1606.07898 [hep-ex].

[10] M. B. Voloshin and L. B. Okun, JETP Lett. 23, 333 (1976) [Pisma Zh. Eksp. Teor. Fiz. 23, 369 (1976)];

[11] N. A. Tornqvist, Phys. Rev. Lett. 67, 556 (1991); N. A. Tornqvist, Z. Phys. C 61, 525 (1994) hep-ph/9310247; N. A. Tornqvist, Phys. Lett. B 590, 209 (2004) hep-ph/0402237.

[12] M. Karliner and H. J. Lipkin, arXiv:0802.0649 [hep-ph]; M. Karliner and J. L. Rosner, Phys. Rev. Lett. 115 (2015) no.12, 122001 arXiv:1506.06386 [hep-ph]]; M. Karliner, Acta Phys. Polon. B 47, 117 (2016).

[13] C. E. Thomas and F. E. Close, Phys. Rev. D 78, 034007 (2008) arXiv:0805.3653 [hep-ph]]; F. Close, C. Downum and C. E. Thomas, Phys. Rev. D 81, 074033 (2010) arXiv:1001.2553 [hep-ph]].

[14] S. Ohkoda, Y. Yamaguchi, S. Yasui, K. Sudoh and A. Hosaka, Phys. Rev. D 86, 034019 (2012) arXiv:1202.0760 [hep-ph]]; S. Ohkoda, Y. Yamaguchi, S. Yasui, K. Sudoh and A. Hosaka, arXiv:1209.0144 [hep-ph]. 
[15] M. T. AlFiky, F. Gabbiani and A. A. Petrov, Phys. Lett. B 640, 238 (2006) hep-ph/0506141]; I. W. Lee, A. Faessler, T. Gutsche and V. E. Lyubovitskij, Phys. Rev. D 80, 094005 (2009) arXiv:0910.1009 [hep-ph]]; M. Suzuki, Phys. Rev. D 72, 114013 (2005) hep-ph/0508258]; J. R. Zhang, M. Zhong and M. Q. Huang, Phys. Lett. B 704, 312 (2011) arXiv:1105.5472 [hep-ph]]; D. V. Bugg, Europhys. Lett. 96, 11002 (2011) arXiv:1105.5492 [hep-ph]]; J. Nieves and M. P. Valderrama, Phys. Rev. D 84, 056015 (2011) arXiv:1106.0600 [hep-ph]]; M. Cleven, F. K. Guo, C. Hanhart and U. G. Meissner, Eur. Phys. J. A 47, 120 (2011) arXiv:1107.0254 [hep-ph]]; T. Mehen and J. W. Powell, Phys. Rev. D 84, 114013 (2011) arXiv:1109.3479 [hep-ph]]; X. W. Kang, Z. H. Guo and J. A. Oller, Phys. Rev. D 94 (2016) no.1, 014012 arXiv:1603.05546 [hep-ph]].

[16] E. S. Swanson, Phys. Rept. 429, 243 (2006) hep-ph/0601110; Z. F. Sun, J. He, X. Liu, Z. G. Luo and S. L. Zhu, Phys. Rev. D 84, 054002 (2011) arXiv:1106.2968 [hep-ph]];

[17] A. V. Manohar and M. B. Wise, Nucl. Phys. B 399, 17 (1993) hep-ph/9212236; N. Brambilla et al., Eur. Phys. J. C 71, 1534 (2011) arXiv:1010.5827[hep-ph]]; M. B. Voloshin, Prog. Part. Nucl. Phys. 61, 455 (2008) arXiv:0711.4556 [hep-ph]]; J. M. Richard, arXiv:1606.08593 [hep$\mathrm{ph}]$.

[18] D. O. Riska and N. N. Scoccola, Phys. Lett. B 299, 338 (1993).

[19] M. A. Nowak, I. Zahed and M. Rho, Phys. Lett. B 303, 130 (1993).

[20] S. Chernyshev, M. A. Nowak and I. Zahed, Phys. Rev. D 53, 5176 (1996) hep-ph/9510326.

[21] M. Nielsen, F. S. Navarra and S. H. Lee, Phys. Rept. 497, 41 (2010) arXiv:0911.1958 [hep-ph]].

[22] Y. Liu and I. Zahed, arXiv:1608.06535 [hep-ph].

[23] G.E. Brown and A.D. Jackson, The nucleon-nucleon interaction, Ed. North Holland (1976).

[24] A. R. Edmonds, "Angular momentum in quantum mechanics," Ed. Princeton Univ. Press (1974).

[25] K. S. Thorne, Rev. Mod. Phys. 52, 299 (1980).

[26] M. A. Nowak and I. Zahed, Phys. Rev. D 48, 356 (1993).

[27] M. Karliner and J. L. Rosner, arXiv:1601.00565 [hep-ph]. 